\title{
An RCT to evaluate the utility of a clinical protocol for staff in the management of behavioral and psychological symptoms of dementia in residential aged-care settings
}

\author{
Marita P. McCabe ${ }^{\mathrm{a} *}$, Michael Bird ${ }^{\mathrm{b}}$, Tanya E. Davison ${ }^{\mathrm{c}}$, David Mellor ${ }^{\mathrm{a}}$, Sarah MacPherson ${ }^{\mathrm{d}}$, David Hallford ${ }^{\mathrm{a}}$ and \\ Melissa Seedy ${ }^{\mathrm{a}}$ \\ ${ }^{a}$ School of Psychology, Deakin University, Victoria, Australia; ${ }^{b}$ Dementia Services Development Centre, Bangor University, Bangor, \\ North Wales, UK; ${ }^{c}$ Aged Mental Health Research Unit, School of Psychology \& Psychiatry, Monash University, Victoria, Australia; \\ ${ }^{d}$ Aged Care Evaluation Unit, Southern LHD, Queanbeyan, Australia
}

(Received 16 May 2014; accepted 3 September 2014)

\begin{abstract}
Objectives: Behavioral and psychological symptoms of dementia (BPSD) cause significant stress and distress to both agedcare residents and staff. This study evaluated a training program to assist staff to manage BPSD in residential care.

Method: A randomised controlled trial (RCT) was employed. The study was included in the Australian and New Zealand Clinical Trial Register residential care facilities. Staff $(n=204)$ and residents $(n=187)$ were from 16 residential care facilities. Facilities were recruited and randomly assigned to four staff training conditions: (1) training in the use of a BPSD-structured clinical protocol, plus external clinical support, (2) a workshop on BPSD, plus external clinical support, (3) training in the use of the structured clinical protocol alone, and (4) care as usual. Staff and resident outcome measures were obtained pre-intervention, three months and six months post-intervention. The primary outcome was changes in BPSD, measured using the Cohen-Mansfield Agitation Inventory (CMAI) as well as frequency and duration of challenging behaviors. Secondary outcomes were changes in staff adjustment.

Results: There were improvements in challenging behaviors for both intervention conditions that included training in the BPSD instrument, but these were not maintained in the condition without clinical support. The training/support condition resulted in sustained improvements in both staff and resident variables, whereas the other conditions only led to improvement in some of the measured variables.

Conclusion: These results demonstrate the effectiveness of the BPSD protocol in reducing BPSD and improving staff selfefficacy and stress.
\end{abstract}

Keywords: BPSD; staff training program; effectiveness of intervention; role of clinical support

\section{Introduction}

Behavioral and psychological symptoms of dementia (BPSD) present a major clinical issue in the care of the elderly. Symptoms such as aggression, shouting, repetitive questions, and sexual disinhibition are particularly challenging for caregivers (Visser et al., 2008). Together with incontinence, these symptoms cause distress to family members and are one factor that leads to family members deciding to move older people into residential facilities, where prevalence of challenging behavior is also high (Seitz, Purandare, \& Conn, 2010; Wetzels, Zuidema, de Jonghe, Verhey, \& Koopmans, 2010). Such challenging behaviors are associated with stress and burnout among care staff (Davison, Hudgson, McCabe, George, \& Buchanan, 2006), many of whom in Australia, Sweden, and United Kingdom are poorly paid and insufficiently trained and supported (Edberg et al., 2008). Furthermore, more than any other clinical issue, they lead to aged-care staff requesting assistance from mental health services (Draper, Meares, \& McIntosh, 1998). New strategies to improve staff management of challenging behavior are required. This paper evaluates a staff training program to assist staff to implement a structured clinical protocol to determine the probable causes of BPSD exhibited by each resident, and adopt strategies to manage those causes which are available to intervention.

Recent guidelines, including the International Psychogeriatric Association Complete Guide to BPSD (International Psychogeriatric Association [IPA], 2012) acknowledge multiple etiologies for BPSD, including genetic, neurobiological, psychosocial, medical, and physical factors. Given such a complex causal mix, it follows that there will be wide variability between cases even if the behavior is the same. Addressing case-specific causal factors (that is, using a personalized individual approach) rather than applying standard treatments would therefore appear to be an appropriate approach. Genetic and neurobiological variables may not be currently treatable, but many psychosocial and physical/medical factors are potentially malleable. For example, many interacting factors can contribute to vocal disruption in dementia, including pain or depression (Cohen-Mansfield \& Werner, 1999), the way care is carried out (Bird \& Blair, 2010), sensory loss (Hallberg, Norberg, \& Erikson, 1990), overstimulation (Meares \& Draper, 1999), or loneliness (Hallberg, Edberg, Nordmark, \& Johnsson, 1993).

It follows that applying a standard intervention aimed only at the behavior, rather than its potential causes, and

*Corresponding author. Email: marita.mccabe@deakin.edu.au 
why it is perceived to be a problem, is a limitation of therapeutic options. It also means that treatable causes of resident suffering, and so their expressed distressed behavior, often remain unaddressed. For example, Elmstahl, Stenberg, Annerstedt, and Ingvad (1998) found that nursing home residents with dementia who were in pain were more likely to be on anti-psychotics than analgesics.

A surprisingly small number of trials have taken a case-specific approach to causal factors. These trials have used mainly psychosocial methods, but some studies have also included pharmacological interventions. In the main, these case-specific studies have had more rigorous methodology than trials of standardized psychosocial interventions, and have produced superior results. These include reductions in frequency and perceived severity of the target behavior and GP call outs (Davison et al., 2006); reductions in anti-psychotic use (Fossey et al., 2006); reductions in agitated behavior and increases in observed participant pleasure (Cohen-Mansfield, Libin, \& Marx, 2007); and reductions in BPSD frequency and perceived severity, staff stress, hospitalizations, anti-psychotic use, and drug side effects (Bird, Llewellyn Jones, \& Korten, 2009; Bird, Llewellyn Jones, Korten, \& Smithers, 2007). Brechen, Murphy, James, and Codner (2013) highlight the need to examine psychological interventions as alternatives to anti-psychotic medication for the treatment of BPSD. They argue for a stepped care model of assessment and intervention. This study was designed to evaluate the effectiveness of these high intensity interventions outlined by Brechen et al. (2013). These interventions are focused specifically on a case-specific approach to determine the factors related to BPSD and implement appropriate interventions, primarily from a psychosocial perspective. The approach in this paper is focused on step 3 [after recognition (step 1) and low intensity interventions (step 2)].

Based on the above literature, in consultation with medical and nursing colleagues, and following a pilot trial, we developed a structured clinical protocol using a checklist format. This check-list helped staff to assess risk and to take them systematically through the most common causes of challenging behavior (that is, behavior that distresses residents, staff, and/or others). The screening process starts with medical factors, then pain and discomfort, then mental illness, especially depression, then level of staff stress and staff perception, followed by the care environment, including social interactions. The structured clinical protocol is intended to achieve the following.

- Educate staff about the fact that challenging behavior usually has causes, what those causes might be, and assist them to develop emotional distance to assess it.

- Empower staff to assess and manage more cases themselves, or even pre-empt problems by becoming more literate about common causal factors and improving care.

- Reduce reliance on outside clinicians with the necessary expertise - a scarce and time-limited resource.

- If outside assistance is required, enable senior staff to provide in-coming clinicians, including GPs, with much more detail across many more domains than can be gleaned in what is commonly accessed in a single hurried visit.

Further information about the clinical protocol is available from the first author on request.

Our hypothesis was that, because of the need for substantial input to change the clinical culture of residential care facilities, only the training/support and support conditions would be superior to the control group, but that training/support which included the protocol would be superior to support in improving both resident behavior and a range of staff measures (see Method for the four different conditions). The primary outcome measure was changes in BPSD and the secondary outcome measures were changes in staff adjustment.

\section{Method \\ Participants}

\section{Residents}

Aged-care residents were recruited from 16 residential agedcare facilities. Only facilities with more than 60 residents were recruited and most residents in these facilities experienced dementia. Residents were identified by senior staff as suitable for the study, if they had a diagnosis of dementia (MMSE [mini mental status examination] < 26) and significant challenging behavior as reported by a staff member. Consent was provided through residents' relatives who received letters detailing the study. Of the 248 residents initially identified, consent was gained for 214 (response rate of $87 \%$ ). In addition to an inability to contact next of kin in a small number of cases, reasons for non-consent/non-participation were: uncomfortable with the research, lack of understanding, and language barriers. Accounting for resident deaths or removal from facilities during the course of the study, the final sample (i.e., residents for whom data were collected for at least two time points) comprised 187 individuals: 134 women and 53 men ranging in age from 48.6 to 98.0 years $(M=83.0, S D=8.8)$. Residents' level of psychotropic medication was not altered for the duration of the study. At baseline, $73(39 \%)$ of the residents were on anti-psychotic medication, $38(20 \%)$ were on antidepressants, and $86(46 \%)$ were on pain medication.

\section{Staff}

The primary point of contact at each facility was asked to recruit and obtain consent from staff members from each of the 16 facilities. Consent was gained from 261 staff members. The final sample for which data were collected for at least two time points comprised 177 women and 27 men ranging in age from 16.9 to 68.0 years $(M=43.0$, $S D=13.4)$. Of these, 127 were personal care assistants (training was a basic certificate in aged care), 48 registered or enrolled nurses, and 29 individuals classified as 'other' (e.g., lifestyle assistants, diversional therapists). Staff had been working in aged care for an average of 9.3 $(S D=8.7)$ years, and over the 12 months preceding their 
involvement in the study had received an average of 8.2 $(S D=13.0)$ hours of dementia-specific training. The main reason for staff attrition was ceasing employment at the facility.

\section{Materials}

\section{Residents and behavior}

Demographic data, including age, gender, time residing at the facility, dementia diagnosis, and other relevant diagnoses, were obtained from the residents' medical file at baseline. The severity/stage of dementia was also assessed using the Clinical Dementia Rating (CDR) (Hughes, Berg, Danziger, Coben, \& Martin, 1982; Morris, 1993), which assesses six domains (memory, orientation, judgment, community affairs, home and hobbies, and personal care). The scale was completed collaboratively by consensus of a minimum of two staff members for each resident at baseline and six months post-intervention. An overall CDR score was derived using a standard algorithm, available at http://www.biostat.wustl.edu/ adrc/cdrpgm/ index.html, with total scores of 0 representing no dementia and $0.5,1,2$, and 3 indicating questionable, mild, moderate, and severe dementia, respectively (Morris, 1993). Using additional rating criteria, scores of 4 and 5 representing profound and terminal dementia, respectively (Dooneief, Marder, Tang, \& Stern, 1996), were also included.

The most problematic behavior for each resident and the time of day at which it typically occurred were initially identified by at least two staff members closely involved with the resident's care. Data on actual frequency or duration (for high frequency behaviors) were collected with the assistance of staff by a research assistant blind to the research design, as unobtrusively as possible over a one-week period at each facility for all three time points (pre-intervention, three months and six months post-intervention). Where possible, the behavior was monitored at the same time of day across time points and typically involved two to three hours of direct observation per resident by the researcher. For some cases, particularly those with less frequently occurring behaviors, staff kept records of observations throughout a specified time period, such as during their shift or over 48 hours, as determined most appropriate by the researcher.

The 14-item version of the Cohen-Mansfield Agitation Inventory (CMAI) Cohen-Mansfield, 1986), also completed by at least two staff concurrently, measured the frequency with which agitated behaviors manifested over the previous two-week period. Responses were recorded on a seven-point Likert scale from 1 (never) to 7 (several times an hour). This measure of challenging behavior used by staff in their day-to-day work with residents and good inter-rater reliability has been reported for this measure (Werner, Cohen-Mansfield, Koroknay, \& Braun, 1994). In this study, internal reliability was good $(\alpha=.79)$. These data were collected on all residents at pre-intervention, three months and six months post-intervention.

\section{Staff reactions and perceptions}

Staff stress associated with each resident's behavior was assessed using the Carer Stress Scale. Two to three staff familiar with each resident rated how much stress each resident's identified behavior caused them personally from 1 (no stress) to 5 (extremely stressful), and the average score at each time point was then calculated. The disruptiveness of each resident's behavior was also assessed with a similar single question five-point scale, with staff taking into account the disruption caused to other residents, staff time required to manage the behavior, and the effects on visitors and staff. Good test-retest reliability has been demonstrated for this instrument (Bird et al., 2007).

\section{Other staff measures}

Demographic data, including age, gender, and years working in aged care, were obtained at baseline from each participating staff member.

General strain, not related to specific residents, was assessed using the 29-item Strains in Dementia Care Scale - SDCS (Edberg et al., 2008). The SDCS contains items relating to frustrated empathy, difficulty in understanding residents, balancing competing needs, balancing emotional involvement with residents, and perceived lack of appreciation from others. Staff rated on a four-point scale how frequently a situation or feeling related to care of residents was experienced, from 1 (never/rarely) to 4 (very often), and how much stress it caused when it did occur, from 1 (none/hardly any) to 4 (high stress). A total score was calculated for frequency of strains and stress associated with these strains. Internal reliability was high in this study, at $\alpha=.90$ for the total frequency score and $\alpha=.95$ for the total stress score.

The Approaches to Dementia Questionnaire (ADQ; Lintern, Woods, \& Phair, 2000) assesses staff attitudes towards people with dementia. Level of agreement is obtained on a five-point Likert scale ranging from 1 (strongly agree) to 5 (strongly disagree) on 19 statements about people with dementia and their care. A total score is derived, with higher scores indicating a more positive attitude towards caring for individuals with dementia. The ADQ has demonstrated good reliability and validity measured against observed staff behavior (Lintern, 2001). Internal reliability in this study was found to be acceptable $(\alpha=.81)$.

The self-efficacy of dementia care is a self-report instrument containing six statements about staff members' perceptions of their skills and confidence in providing dementia care, including managing challenging behaviors. Items are rated on a five-point Likert response format ranging from 1 (strongly disagree) to 5 (strongly agree). Previous studies have reported good reliability for this scale (Davison et al., 2007), and it was found to be adequate in this study $(\alpha=.67)$.

CDR was completed at Time 1 and Time 3 only. All other resident and staff outcome measures were completed 
at baseline (T1), three months (T2), and six months postintervention (T3). Where possible, the same staff completed measures on each resident across the three time points, but practical issues (e.g., staff turnover, rostering) sometimes prevented this. All outcome data were collected by research assistants blind to the study condition.

\section{Procedure}

Ethics approval for the study was obtained from the University Ethics Committee.

We conducted a randomized controlled trial to evaluate the effectiveness of training staff in using this structured clinical protocol with residents identified as manifesting BPSD. The study has been included on the Australian and New Zealand Clinical Trial Register. We were interested in whether this approach provided further benefit beyond the common method of training staff in person-centered care. We also aimed to determine whether additional clinical support would be required for staff to successfully implement the protocol. Accordingly, our design involved the following four conditions, over two waves and with data collected at baseline, three months, and six months after the training program.

(1) Staff workshop on BPSD and person-centered care, plus a training session on the structured clinical protocol, and fortnightly clinical support visits for three months from an experienced mental health clinician to assist staff in the use of the protocol with identified residents (training/support condition).

(2) Staff workshop on person-centered care and BPSD, plus clinical support visits to assist with applying person-centered care to identified residents for the same period as the condition. This condition was called the support condition.

(3) Staff training in the use of the structured clinical protocol, but no clinical support. This condition was called the training condition.

(4) Control condition: care as usual condition.

\section{Recruitment}

Sixteen Australian residential aged-care facilities were recruited to participate in the study, eight from the Australian Capital Territory/New South Wales and eight from Victoria, across two consecutive phases of data collection. All facilities that were approached to participate in the study agreed to participate. Facilities were randomized to one of the four study/intervention conditions using a cluster randomized controlled design (i.e., the facility rather than the participants/residents or staff were the unit of randomization). Randomization occurred by facilities being allocated to one of the conditions as they were recruited into the study. The numbers $1,2,3$, and 4 were placed in a box (in each of the two locations) in both year 1 and year 2 . The number that was drawn out for the facility determined which of the four conditions the facility was allocated to.
Aged-care residents were recruited through consultation with senior staff at each facility. The inclusion criteria required a positive diagnosis of dementia, and the presence of at least one challenging behavior, defined as 'any behavior associated with dementia which causes distress or danger to the person with dementia and/or others' (Bird et al., 2009). The sample size was determined to provide sufficient power to conduct the analyses with a small effect size.

\section{Intervention}

A two-hour training session in which staff were helped to work through and identify probably causal factors for the behavior of residents, and develop potential ways of ameliorating these causes (training/support and training conditions).

\section{Educational workshop}

A two-hour workshop providing an overview of dementia, BPSD, and person-centered care strategies was provided (training/support and support conditions).

\section{Clinical support}

A mental health professional experienced in working with BPSD provided clinical support for training/support and support conditions. For the training/support condition, this focused on assisting staff in implementing the structured clinical protocol to manage the BPSD of the residents participating in the study. For the support condition, this involved providing resident-specific psycho-educational support to staff, with a focus on person-centered care strategies. Clinical support was provided over six fortnightly sessions of two hours each across the first 12 weeks of the study.

\section{Data analytic strategy}

All data were analyzed using IBM SPSS 20.0. The original power calculation of 0.8 was based on a sample size of 50 residents and 50 staff in each group with the expectations of a moderate effect size. We included all participants who provided data at baseline (Time 1) and at least one post-baseline time point. As scores derived from resident behavioral monitoring data were based on different metrics (i.e., frequency or duration) and varied greatly in absolute number (e.g., 150 counts of repetitive questioning over one hour compared to three counts of physical aggression across several days), percentage change scores from baseline (T1) to three-month follow-up (T2) and from baseline to six-month follow-up (T3) were calculated for each resident. Three-month change scores were the difference between three-month and baseline scores divided by baseline scores. Six-month change scores were the differences between six-month scores and baseline scores divided by baseline scores. Positive values indicate improvement (with 100\% change representing complete cessation of the target behavior) and negative values 


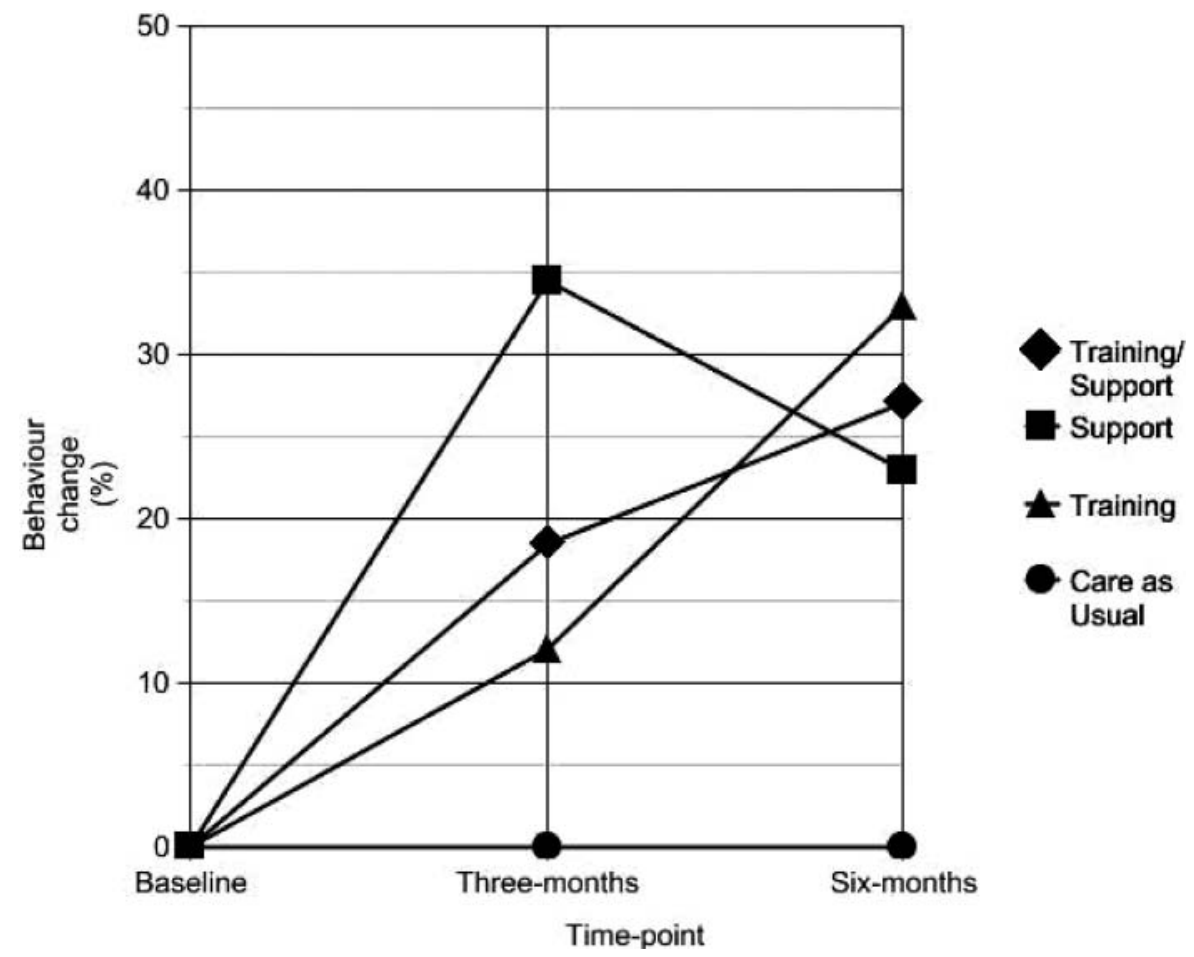

Figure 1. Monitored behavior change over time-points. Higher scores denote improvements in challenging behavior of resident, with $100 \%$ indicating complete cessation of target behavior.

indicate worsening of the target behavior. The median of resident change scores was used to represent the overall percentage of change in each condition (see Figure 1). The CMAI scores were also converted into percentage change scores so that comparisons could be made between the two measures of challenging behavior.

Repeated measures analysis of variance (ANOVAs) were conducted for each outcome measure over time in each condition, using Bonferroni corrections to control the family-wise error rate of each group of tests at $p<.05$.

\section{Results}

\section{Residents}

Descriptive statistics for the resident sample are provided in Table 1. Univariate tests indicated that the conditions differed significantly on baseline CDR scores, $F(3$, $183)=3.06, p<.05$, with residents in the training condition having significantly higher baseline CDR scores than those in the control condition $(p<.05, d=.61)$. No significant differences were found for age, years in facility, or six-month CDR rating. Paired sample $t$-tests showed that the CDR ratings increased over time in the care as usual condition, $t(34)=-2.25, p<.05, d=.34$.

\section{Staff}

Descriptive statistics for the staff sample are provided in Table 2. Univariate tests indicated that the conditions differed significantly on number of years working in aged care, $F(3,198)=4.28, p<.01$, with staff in the training/ support condition working more years in aged care than those in the care as usual condition $(p<.05, d=.44)$. No other significant differences were found.

\section{Challenging behavior}

Results from direct behavior monitoring indicated that the main identified behavior improved in all intervention conditions but not in the control condition. In the training/ support condition, median improvements were $18.5 \%$

Table 1. Demographic data for aged-care resident sample.

\begin{tabular}{lccccc}
\hline & Training/Support & Support & Training & Care as usual & Total \\
\hline No. of residents & 53 & 49 & 48 & 37 & 187 \\
Age $(S D)$ & $84.74(6.85)$ & $82.71(9.20)$ & $82.85(8.45)$ & $81.25(11.03)$ & $83.03(8.83)$ \\
Females (\%) & $38(72 \%)$ & $36(74 \%)$ & $34(71 \%)$ & $26(70 \%)$ & $134(72 \%)$ \\
Years in facility $M(S D)$ & $2.13(2.06)$ & $2.64(2.35)$ & $1.86(1.66)$ & $2.54(1.97)$ & $2.27(2.04)$ \\
Baseline CDR $M(S D)$ & $2.64(0.6)$ & $2.74(.88)$ & $3.06(1.26)$ & $2.43(.73)$ & $2.73(1.01)$ \\
Six-month CDR $M(S D)$ & $2.70(0.77)$ & $2.87(0.87)$ & $3.07(1.16)$ & $2.71(.91)$ & $2.85(0.91)$ \\
\hline
\end{tabular}

Note: $\mathrm{CDR}=$ Clinical Dementia Rating 
Table 2. Demographic data for aged-care staff sample.

\begin{tabular}{|c|c|c|c|c|c|}
\hline & Training/Support & Support & Training & Care as usual & Total \\
\hline No. of staff & 50 & 51 & 49 & 54 & 204 \\
\hline Age $M(S D)$ & $45.5(12.2)$ & $41.6(14.2)$ & $44.5(14.0)$ & $40.8(13.1)$ & $43.0(13.4)$ \\
\hline Females $(\%)$ & $47(94 \%)$ & $48(94.1 \%)$ & $42(85.7 \%)$ & $40(74.1 \%)$ & $177(86.8 \%)$ \\
\hline PCA & 26 & 42 & 28 & 31 & 127 \\
\hline Nurses & 14 & 5 & 17 & 12 & 48 \\
\hline Other & 10 & 4 & 4 & 11 & 29 \\
\hline Years in aged care $M(S D)$ & $12.0(9.7)$ & $8.0(7.3)$ & $10.9(10.2)$ & $6.7(6.3)$ & $9.3(8.67)$ \\
\hline Training $M(S D)$ & $11.5(14.5)$ & $7.0(11.6)$ & $6.1(7.0)$ & $8.2(15.9)$ & $8.2(13.0)$ \\
\hline
\end{tabular}

Note: PCA = personal care assistants, Training = hours of completed dementia training in last 12 months

(T1-T2) and 27\% (T1-T3); in the support condition, they were $34.5 \%(\mathrm{~T} 1-\mathrm{T} 2)$ and $23 \%(\mathrm{~T} 1-\mathrm{T} 3)$; in the training condition, they were $12 \%(\mathrm{~T} 1-\mathrm{T} 2)$ and $33 \%$ (T1-T3); and in the care as usual condition, they were $0 \%(\mathrm{~T} 1-\mathrm{T} 2)$ and $0 \%(\mathrm{~T} 1-\mathrm{T} 3)$. Figure 1 shows percentage changes in the monitored behavior in each condition from baseline to three months and baseline to six months.

On the CMAI, significant time effects for challenging behavior were found for the training/support condition, $F$ $(2,104)=5.28, p<.05$, partial $\eta^{2}=.09$, with follow-up $t$-tests showing significant reductions from $\mathrm{T} 1$ to $\mathrm{T} 2, t(1$, $52)=2.48, p<.05, d=.31$, and $\mathrm{T} 1$ to $\mathrm{T} 3, t(1,52)=2.7$, $p<.01, d=.34$. No significant time effect was found in

Table 3. Means (standard deviations) for frequency of strains, total stress, and self-efficacy of dementia care.

\begin{tabular}{lccc}
\hline & Time 1 & Time 2 & Time 2 \\
\hline Frequency of stains & & & \\
$\quad$ Training/support & $2.32(.56)$ & $2.12(.54)$ & $2.02(.54)$ \\
$\quad$ Support & $2.02(.63)$ & $1.92(.58)$ & $1.92(.56$ \\
Training & $2.09(.60)$ & $1.91(.68)$ & $1.92(.65)$ \\
Case as usual & $1.95(.63)$ & $1.98(.64)$ & $2.03(.69)$ \\
Total stress & & & \\
$\quad$ Training/support & $2.98(1.02)$ & $2.55(.72)$ & $2.21(.87)$ \\
$\quad$ Support & $2.873(.85)$ & $2.51(.68)$ & $2.47(.79)$ \\
$\quad$ Training & $2.24(.84)$ & $2.02(.81)$ & $2.07(.78)$ \\
Care as usual & $2.64(.68)$ & $2.80(.84)$ & $2.64(.82)$ \\
Self-efficacy of dementia & & & \\
$\quad$ Training/support & $3.35(.55)$ & $3.54(.61)$ & $3.56(.61)$ \\
$\quad$ Support & $3.26(.50)$ & $3.36(.48)$ & $3.42(.54)$ \\
$\quad$ Training & $3.39(.49)$ & $3.65(.56)$ & $3.65(.50)$ \\
Care as usual & $3.28(.52)$ & $3.31(.49)$ & $3.32(.60)$ \\
\hline
\end{tabular}

the support condition. In the training condition, significant time effects were found, $F(2,94)=5.71, p<.05$, partial $\eta^{2}=.11$, with follow-up $t$-tests showing significant reductions from $\mathrm{T} 1$ to $\mathrm{T} 2, t(1,47)=3.43, p<.01, d=.47$, but not from $\mathrm{T} 1$ to $\mathrm{T} 3$. In the care as usual condition, significant time effects were found, $F(2,72)=4.47, p<.05$, partial $\eta^{2}=.11$, with follow-up $t$-tests showing no significant change from $\mathrm{T} 1$ to $\mathrm{T} 2$, but significant reductions from $\mathrm{T} 1$ to $\mathrm{T} 3, t(1,36)=2.13, p<.05, d=.38$. An analyses of covariance analysis (ANCOVA) to assess for differences between the training/support and care as usual conditions showed no significant difference (see Table 4).

\section{Staff reactions and perceptions}

For carer stress, significant time effects were found for the training/support condition, $F(2,104)=18.07, p<.01$, partial $\eta^{2}=.26$, with follow-up $t$-tests showing significant reductions in stress from $\mathrm{T} 1$ to $\mathrm{T} 2, t(1,52)=3.13, p<$ $.01, d=.49$, and from $\mathrm{T} 1$ to $\mathrm{T} 3, t(1,52)=5.34, p<.001$, $d=.81$. In the support condition, significant time effects were also found, $F(2,96)=5.97, p<.05$, partial $\eta^{2}=$ .11 , with follow-up $t$-tests showing significant reductions from $\mathrm{T} 1$ to $\mathrm{T} 2, t(1,48)=2.59, p<.05, d=.42$, and from $\mathrm{T} 1$ to $\mathrm{T} 3, t(1,48)=3.16, p<.01, d=.44$. No significant time effect was found for either the training only or the care as usual conditions. An ANCOVA to assess for differences between the training/support and support conditions showed that at T3 staff in the training/support condition reported significantly lower stress than staff in the support only condition, $F(2,99)=4.51, p<.05$ (see Table 3 ).

For staff-perceived disruption, a similar pattern of outcomes was found. Effects for time were found in the training/support condition, $F(2,104)=10.79, p<.01$, partial $\eta^{2}=.17$, with follow-up $t$-tests showing significant

Table 4. Means, standard deviations, significant changes, and effect sizes on the CMAI.

\begin{tabular}{|c|c|c|c|c|c|c|c|}
\hline Condition & Baseline & Three-month & Effect size & Six-month & Effect size & Sig. T1-T3 & Effect size \\
\hline Condition 1 & $3.34(1.01)$ & $3.02(1.07)^{*}$ & $d=.31$ & $2.98(1.08)$ & & Yes* $^{*}$ & $d=.34$ \\
\hline Condition 2 & $3.24(.67)$ & $3.17(.85)$ & & $3.20(.91)$ & & No & \\
\hline Condition 3 & $2.73(.85)$ & $2.33(.86)^{* *}$ & $d=.47$ & $2.47(.96)$ & & No & \\
\hline Condition 4 & $3.20(1.09)$ & $3.22(1.06)$ & & $2.81(.92)^{*}$ & $d=.41$ & Yes* $^{*}$ & $d=.38$ \\
\hline
\end{tabular}

Note: ${ }^{*} p<0.05,{ }^{* *} p<0.01$. 
reductions from $\mathrm{T} 1$ to $\mathrm{T} 2, t(1,52)=3.04, p<.01, d=$ .45 , and from $\mathrm{T} 1$ to $\mathrm{T} 3, t(1,52)=4.3, p<.001, d=.61$. In the support condition, significant time effects were also found, $F(2,96)=8.11, p<.01$, partial $\eta^{2}=.15$, with follow-up $t$-tests showing significant reductions from $\mathrm{T} 1$ to $\mathrm{T} 2, t(1,48)=2.88, p<.01, d=.40$, and from $\mathrm{T} 1$ to $\mathrm{T} 3, t$ $(1,48)=3.63, p<.01, d=.54$. No significant time effect was found for either the training only or the care as usual conditions. An ANCOVA to assess for differences between the training/support and support only conditions showed there was no significant difference.

\section{Other staff measures}

On SDCS, significant time effects were found in the training/support condition on the total frequency (of strains) scale, $F(2,98)=9.49, p<.01$, partial $\eta^{2}=.16$, with follow-up $t$-tests showing no significant change in strain frequency from $\mathrm{T} 1$ to $\mathrm{T} 2$, but significant reductions from $\mathrm{T} 1$ to T3, $t(1,49)=4.63, p<.001, d=.50$. Significant time effects were also found in the training/support condition on the total stress (associated with strains) scale, $F(2,98)$ $=9.91, p<.01$, partial $\eta^{2}=.17$, with follow-up $t$-tests showing significant reductions from $\mathrm{T} 1$ to $\mathrm{T} 2, t(1,49)=$ 2.53, $p<.05, d=.36$, and from $\mathrm{T} 1$ to $\mathrm{T} 3, t(1,49)=3.91$, $p<.001, d=.54$. No significant time effects on the SDCS (frequency of strains or stress associated with strains) were found in frequency or stress for the support only or care as usual conditions, or for frequency for the support only condition.

On ADQ, no significant time effect was found for any of the groups.

On the self-efficacy of dementia care, a significant time effect was found in the training/support condition, $F(2,98)=6.26, p<.05$, partial $\eta^{2}=.11$, with follow-up $t$-tests showing a significant increase in self-efficacy from $\mathrm{T} 1$ to $\mathrm{T} 2, t(1,49)=-2.28, p<.01, d=.32$, and from $\mathrm{T} 1$ to $\mathrm{T} 3, t(1,49)=-2.93, p<.01, d=.36$. No significant time effect was found for the support condition. A significant time effect was found in the training condition, $F(2$, $96)=9.14, p<.01$, partial $\eta^{2}=.16$, with follow-up $t$ tests showing a significant increase from T1 to T2, $t(1$, $48)=-3.1, p<.01, d=.49$, and from $\mathrm{T} 1$ to $\mathrm{T} 3, t(1,48)$ $=-4.04, p<.001, d=.53$. No significant time effect was found for the care as usual condition. An ANCOVA to assess for differences between the training/support and training only conditions showed no significant difference.

\section{Discussion}

The results demonstrate that a structured clinical protocol aimed at helping staff identify and address the individual causal factors behind challenging behavior for each resident was effective across all but one measure when combined with clinical support. It reduced the frequency of the referred behavior, its perceived disruptiveness, staff stress related to the behavior, and CMAI frequency, as well as more generic staff measures: frequency and degree of general strain, and sense of self-efficacy in dementia care. The effects were apparent after three months, at which point clinical support was withdrawn but were still maintained three months later. The only measure that did not show improvement was staff attitudes, but this was true for all conditions.

When the same amount of clinical support was provided but without the structured protocol, it reduced staff stress related to the referred behavior and its perceived disruptiveness and these effects were maintained, but it had no effect on the CMAI frequency scores, strain in dementia care, nor self-efficacy. When the protocol was available but clinical support was not offered, there was improvement in behavior (CMAI) up to three months but this was not maintained. There was sustained improvement in staff self-efficacy in dementia care - including ability to manage challenging behavior. Training in the clinical protocol alone without any clinical support had no effect on any other measure.

All three active conditions showed improvement in frequency of the referred behavior, obtained through behavior monitoring. The care as usual condition, which received neither the training in the clinical protocol nor clinical support, showed no change in the referred behavior or any staff measure but, unexpectedly, did show improvement in the CMAI frequency from T2 to T3. Despite this improvement, which occurred without any input, it is reasonable to infer that the improvements which occurred in the three active conditions were due to the interventions. However, there is always the possibility that simply being in an intervention (whatever the nature of the intervention) was responsible for the improvement in the CMAI scores.

Given that behavior becomes challenging because of an interaction between its nature and intensity, and a highly individual and variable staff response to this behavior (Bird et al., 2007), it is to be expected that, following effective clinical support in person centered care, staff will perceive the resident differently. Thus, in the clinical support only condition, staff were less stressed by the behavior, and perceived it as less challenging. In the training only condition, they were given the structured clinical protocol to help them identify and manage causal factors potentially behind the behavior, and it is to be expected that, if they used it as planned, there would be at least a temporary reduction in behavior and an increase in perceived self-efficacy in understanding and dealing with distressed residents. Effect sizes for most of these staff measures were at least medium and these are not trivial findings, especially given that these were very experienced staff. These findings have implications for clinical practice in relation to the management of BPSD.

It is often staff variables which contribute to BPSD and also determine whether or not it is perceived as 'challenging' (Bird et al., 2007; Davison et al., 2006; McCabe, Davison, \& George, 2007). Unless they feel that they have the personal resources, skills, knowledge, and support, staff are likely to experience stress reactions and burnout that can lead to ineffective management of these problems, as well as high levels of staff turnover (Davison et al., 2006). However, as evidenced by the findings of this study, if staff are provided with appropriate training 
in how to manage BPSD, as well as clinical support to embed these practices, this appears to benefit both residents and staff.

The results of this study support the model for treatment of BPSD outlined by Brechen et al. (2013). The first two steps in this model are to identify causes and risks for BPSD, followed by management of the contextual environment within which BPSD occurs. The third step, which is largely evaluated by this study, involves the implementation of interventions specifically designed to the needs to each resident. Through the training in the clinical protocol and the three months of clinical support, this intensely high intervention was provided, so that individualized care was possible.

In order to obtain a more substantial change in the severity of challenging behaviors, it may be necessary to ensure that the use of the structured clinical protocol is embedded into organizational procedures, and is incorporated into the general care regime of residents: for example, on intake and whenever BPSDs occur. Future research needs to explore the role of organizational factors in terms of the adoption of clinical protocol into routine practice. Anecdotal reports from staff suggested there was considerable variation in the degree to which individual facilities within the training/support condition complied with the protocol. If staff were consistently encouraged to use the clinical protocol, and they developed greater confidence in its use - particularly in implementing strategies to address the identified causes of symptoms, the level of BPSD may reduce more substantially. We did not collect measurable data for all facilities on implementation rate or staff turnover, and so it was not possible to include this in the analyses. A limitation of this study was that randomization by facility may have led to systematic differences between the groups, even though these differences were controlled for by the use of analysis of covariance analyses. It is also possible that the staff expectations about the outcome of the study may have impacted their reports of BPSD in terms of their completion of CMAI. However, this is unlikely to be the case for the observation of resident behavior as these observations were collected by research personnel who were blind to the condition to which the facility was allocated.

The presence of barriers, such as a lack of commitment to improving resident mental health within the context of a poorly resourced aged-care sector, and insufficient knowledge about best practice in mental health among facility managers are likely to have limited the implementation of the study design, and so lowered the possible benefits of the use of the clinical protocol. In addition, high staff turnover as well as poor staff motivation and ineffective communication between staff identified in this study may also have acted to prevent the translation of the protocol into the routine care of residents in the facilities. These factors need to be evaluated in future research studies.

Blume, Ford, Baldwin, and Huang (2009) demonstrated that supportive workplace relations are a key factor in the translation of skills into practice, and highlighted the importance of organizational barriers that prevent this transfer from occurring. Further research is necessary to address these organizational barriers to the routine facility wide implementation of the use of the clinical protocol. However, the current findings offer the possibility of an alternative non-pharmacological mechanism to manage challenging behaviors among residents and so restore the well-being of residents and staff.

\section{Acknowledgements}

The study has been included in the Australian and New Zealand Clinical Trial Register: ACTRN1261000189022.

\section{Funding}

This study was supported by a grant from the National Health and Medical Research Council [grant number 594783].

\section{References}

Bird, M., \& Blair, A. (2010). Clinical psychology and anxiety and depression in dementia. Nordic Psychology, 62, 43-54.

Bird, M., Llewellyn-Jones, R., \& Korten, A. (2009). An evaluation of the effectiveness of a case-specific approach to challenging behavior in dementia. Aging \& Mental Health, 13, $73-83$.

Bird, M., Llewellyn Jones, R.H., Korten, A., \& Smithers, H. (2007). A controlled trial of a predominantly psychosocial approach to BPSD: Treating causality. International Psychogeriatrics, 19, 874-891.

Blume, B.D., Ford, K.J., Baldwin, T.T., \& Huang, J.L. (2009). Transfer of training: A meta-analytic review. Journal of Management, 36, 1065-1105.

Brechen, D., Murphy, G., James, I.A., \& Codner, J. (2013). Alternatives to antipsychotic medication: Psychological approaches to managing psychological and behavioural distress in people with dementia. Leicester: British Psychological Society.

Cohen-Mansfield, J. (1986). Agitated behaviors in the elderly. II. Preliminary results in the cognitively deteriorated. Journal of the American Geriatrics Society, 9, 361-381.

Cohen-Mansfield, J., Libin, A., \& Marx, M.S. (2007). Nonpharmacological treatment of agitation: A controlled trial of systematic individualized intervention. Journal of Gerontology, 62A, 908-916.

Cohen-Mansfield, J., \& Werner, P. (1999). Longitudinal predictors of non-aggressive agitated behaviors in the elderly. International Journal of Geriatric Psychiatry, 14, 831-844.

Davison, T., Hudgson, C., McCabe, M., George, K., \& Buchanan, G. (2006). An individualized psychosocial approach for 'treatment resistant' behavioral symptoms of dementia among aged care residents. International Psychogeriatrics, 19, 859-873.

Davison, T.E., McCabe, M.P., Visser, S., Hudgson, C., Buchanan, G., \& George, K. (2007). Controlled trial of dementia training with a peer support group for aged care staff. International Journal of Geriatric Psychiatry, 22, 868-873.

Dooneief, G., Marder, K., Tang, M., \& Stern, Y. (1996). The Clinical Dementia Rating scale: Community-based validation of 'profound' and 'terminal' stages. Neurology, 46, $1746-1749$

Draper, B., Meares, S., \& McIntosh, H. (1998). A psychogeriatric outreach service to nursing homes in Sydney. Australasian Journal on Ageing, 17, 184-186.

Edberg, A-K., Bird, M., Richards, D., Woods, R., Keeley, P., \& Davis-Quarrell, V. (2008). Strain in nursing care of people with dementia: Nurses' experience in Australia, Sweden, and United Kingdom. Aging \& Mental Health, 12, 236-243. 
Elmståhl, S., Stenberg, I., Annerstedt, L., \& Ingvad, B (1998). Behavioral disturbances and pharmacological treatment of patients with dementia in family caregiving: A 2-year follow-up. International Psychogeriatrics, 10, $239-252$.

Fossey, J., Ballard, C., Juszczak, E., James, I.A., Alder, N., Jacoby, R., \& Howard, R. (2006). Effect of enhanced psychosocial care on antipsychotic use in nursing home residents with severe dementia: Cluster randomised trial. British Medical Journal, 332, 756.

Hallberg, I., Norberg, A., \& Erikson, S. (1990). Functional impairment and behavioral disturbances in vocally disruptive patients in psychogeriatric wards compared with controls. International Journal of Geriatric Psychiatry, 5, $53-61$.

Hallberg, I.R., Edberg, A.-K., Nordmark, A., \& Johnsson, K. (1993). Daytime vocal activity in institutionalised severely demented patients identified as vocally disruptive by nurses. International Journal of Geriatric Psychiatry, 8, 155-164.

Hughes, C.P., Berg, L., Danziger, W.L., Coben, L.A., \& Martin, R.L. (1982). A new clinical scale for the staging of dementia. British Journal of Psychiatry, 140, 566-572.

International Psychogeriatric Association (IPA). (2012). Behavioral and psychological symptoms of dementia (BPSD) education pack. Skokiu, IL: Author.

Lintern, T. (2001). Quality in dementia care: Evaluating staff attitudes and behavior (PhD dissertation). University of Wales, Bangor.
Lintern, T., Woods, B., \& Phair, L. (2000). Before and after training: A case study of intervention. Journal of Dementia Care, 8, 15-17.

McCabe, M.P., Davison, T.E., \& George, K. (2007). Effectiveness of staff training programs for behavioral problems among older people with dementia. Aging \& Mental Health, 11, 505-519.

Meares, S., \& Draper, B. (1999). Treatment of vocally disruptive behavior of multi-factorial aetiology. International Journal of Geriatric Psychiatry, 14, 285-290.

Morris, J. (1993). The Clinical Dementia Rating (CDR): Current version and scoring rules. Neurology, 43, 2412-2414.

Seitz, D., Purandare, N., \& Conn, D. (2010). Prevalence of psychiatric disorders among older adults in long-term care homes: A systematic review. International Psychogeriatrics, 22, 1025-1039

Visser, S., McCabe, M.P., Hudgson, C, Buchanan, G., Davison, T., \& George, K. (2008). Managing behavioral symptoms of dementia: Effectiveness of staff education and peer support. Aging \& Mental Health, 12, 47-55.

Werner, P., Cohen-Mansfield, J., Koroknay, V., \& Braun J. (1994). The impact of a restraint reduction program on nursing home residents. Geriatric Nursing, 15, 142-146.

Wetzels, R.B., Zuidema, S.U., de Jonghe, J.F.M., Verhey, R.J., \& Koopmans, R.T.C.M. (2010). Course of neuropsychiatric symptoms in residents with Dementia in nursing home over 2-year period. American Journal of Geriatric Psychiatry, $18,1054-1065$ 